\title{
Fault Diagnosis in a Centrifugal Pump Using Active Magnetic Bearings
}

\author{
Rainer Nordmann and Martin Aenis \\ Mechatronics \& Machine Acoustics, Darmstadt University of Technology, Darmstadt, Germany
}

The number of rotors running in active magnetic bearings (AMBs) has increased over the last few years. These systems offer a great variety of advantages compared to conventional systems. The aim of this article is to use the AMBs together with a developed built-in software for identification, fault detection, and diagnosis in a centrifugal pump. A single-stage pump representing the turbomachines is investigated. During full operation of the pump, the AMBs are used as actuators to generate defined motions respectively forces as well as very precise sensor elements for the contactless measurement of the responding displacements and forces. In the linear case, meaning small motions around an operating point, it is possible to derive compliance frequency response functions from the acquired data. Based on these functions, a model-based fault detection and diagnosis is developed which facilitates the detection of faults compared to state-of-the-art diagnostic tools which are only based on the measurement of the systems outputs, i.e., displacements. In this article, the different steps of the modelbased diagnosis, which are modeling, generation of significant features, respectively symptoms, fault detection, and the diagnosis procedure itself are presented and in particular, it is shown how an exemplary fault is detected and identified.

Keywords Fault diagnosis, Force measurement, Frequency response, Magnetic bearing, Modal analysis, Turbomachinery

In various technical areas, rotating machinery are in operation, such as turbines, pumps, compressors, motors, generators, etc. Users expect that their machines are running safe and reliably and that they have high efficiency and availability. In

Received 22 January 2003; accepted 2 July 2003.

Address correspondence to Martin Aenis, Mechatronics \& Machine Acoustics, Darmstadt University of Technology, Petersenstr. 30, Darmstadt 64287, Germany. E-mail: aenis@mum.tu-darmstadt.de order to satisfy these requirements an integrated failure detection and diagnosis becomes increasingly important for these machines.

The demand on using magnetic bearings in turbomachines has strongly increased over the last five years. This is stated in several recent publications such as Gopalakrishan (1999), Hergt (1999), and in the proceeding of the last magnetic bearing conference (ISMB, 2000). Most importantly here are the active magnetic bearings (AMBs), a typical mechatronic system. Rotors in AMBs already offer a variety of advantages compared to conventional systems. Some of them are the tuning possibilities for stiffness and damping, the absence of wear, the reduction of friction, the high running speeds, and possible unbalance compensation. In various applications the feasibility and profitability of using AMBs in turbomachines have been demonstrated, e.g., Allaire et al. (1989) and McGinnis et al. (1990). However, there is much more potential in such systems than using them as a simple bearing. AMBs also have to be used as sensor and actuator elements. They work in this new generation of turbomachines as an integrated identification and diagnosis tool. In this way it will be possible to design new machines with higher performance, higher reliability, and longer lifetimes.

Equation (1) shows the linear description of the dynamic behavior of a rotor with stiffness, damping, and inertia characteristics, expressed by the matrices $\mathbf{M}, \mathbf{D}$ and $\mathbf{K}$. We assume that the rotor matrices are time-invariant but depend on the running speed and the actual operating condition.

$$
\mathbf{M} \ddot{\mathbf{x}}(t)+\mathbf{D} \dot{\mathbf{x}}(t)+\mathbf{K x}(t)=\mathbf{f}(t)
$$

The forces $\mathbf{f}(\mathrm{t})$ are considered as the system inputs and the displacements $\mathbf{x}(\mathrm{t})$ as the system outputs. When input-output relations are considered in the frequency domain, Equation (1) can be transformed to the following complex frequency response function:

$$
\hat{\mathbf{x}}(\Omega)=\left(\mathbf{K}-\Omega^{2} \mathbf{M}+j \Omega \mathbf{D}\right)^{-1} \hat{\mathbf{f}}(\Omega)=\overline{\mathbf{H}}(\Omega) \hat{\mathbf{f}}(\Omega)
$$

where $\overline{\mathbf{H}}_{k l}(\Omega)$ is the system response (amplitude and phase) of the displacement $\hat{\boldsymbol{x}}_{k}(\Omega)$ due to a unit force excitation $\hat{\mathbf{f}}_{l}(\Omega)$. 
Today, monitoring and diagnosis systems (e.g., Machine Condition Manager ${ }^{\mathrm{TM}} 2000$ from Bently Nevada and VibrotestSerie from Schenck) are not normally an integral component of the turbomachines. These fault detection and diagnosis systems mainly measure the output signals $\mathbf{x}(\mathrm{t})$, the relative and/or absolute motions of the rotor. After signal processing, certain features (threshold values, orbits, frequency spectra, etc.) are created from the measured data. With the deviations of these features from a non-faulty initial state, faults are detected. Subsequently, the diagnosis attempts to recognize possible faults. The difficulty with these procedures is that the causes of the modifications of the output signals cannot be detected clearly. The reason might be due to a change of the process respectively the input, a modification of the system itself, or measurement noise. It should be mentioned that the inputs and outputs of the system are restricted to the degrees of freedom at the bearings (see also Equation (5)).

If AMBs are already integrated in the turbomachines, an improvement of the existing diagnostic techniques can be achieved without any additional hardware installation. AMBs are well suited to operate as sensor elements, being able to measure not only the outputs but also the inputs. And furthermore, AMBs can be used as actuators to excite the system with defined signals, consequently receiving input-output relations. Hence, AMBs are able to measure all three components of Equation (2); the input, the output, and the system characteristics themselves.

The application of AMBs as a force measurement tool to determine hydraulic forces acting on pump rotors was validated by Guinzburg and Buse (1994) and Baun and Flack (1997); both showed good agreements between experimental and theoretical data. Humphris (1992) demonstrated the huge capabilities of an analog-controlled magnetic bearing system with respect to generating diagnostic information. He obtained information about critical speeds, structural resonances, damping factors, and clearance magnitudes. His main purpose, however, was to optimize the behavior of magnetic bearings. Pottie et al. (1994) evaluated measured frequency response functions (FRF) for the identification of fluid-structureinteraction forces. They suggested that a FRF measurement of on-line measured magnetic forces and displacements can potentially be used to perform fault diagnosis. The necessary improvement in the quality of the force measurement to yield accurate FRFs was pointed out by Pottie et al. (1994). Knopf and Nordmann (2001) used the AMBs to identify the rotordynamic coefficients of a turbulent journal bearing with an improved force measurement technique as suggested by Pottie et al. (1994).

In summary, AMBs in turbomachines offer a great potential in obtaining deeper knowledge about the system behavior during operation, which is used for an integrated diagnosis. Adding even more information about the system through modeling, in terms of a-priori knowledge, is a logical consequence and hence performed.

\section{PRINCIPLE OF MODEL-BASED FAULT DIAGNOSIS}

In general, the methods used for fault (fault is any deviation from the normal behavior of the plant) diagnosis are classified into two main groups: those based on mathematical models and those based only on measured signals. Classical diagnostic methods are signal-based, however as Natke and Cempel (1997) already stated: 'A verified and validated mathematical model with sufficiently small errors is the best available knowledge base' (p. 14). Hence, the work presented here concentrates on model-based methods.

The entire model-based diagnosis procedure is split into two tasks: the fault detection and the actual diagnosis (see Figure 1). Again, the faults acting on the system can be divided into two groups: (1) altering the system $\overline{\mathbf{H}}(\Omega)$ and (2) altering the input $\hat{\mathbf{f}}$, respectively the output $\hat{\mathbf{x}}$. These groups are often referred to as multiplicative, respectively additive faults Gertler, 1998). In this article, the focus lies on faults belonging to the first group.

The main steps of the fault detection are:

- Data acquisition and signal processing

- Modeling of the non-faulty plant

- Generation of features

- Generation of symptoms, i.e., residuals

- Quality criterion: no fault/fault detected

The most important part of the entire diagnosis procedure is the generation of features because it is obvious that the results of the diagnosis strongly depend on the information depth contained in the features. The generation of the residuals of the features, specifically the symptoms, is then a comparison between

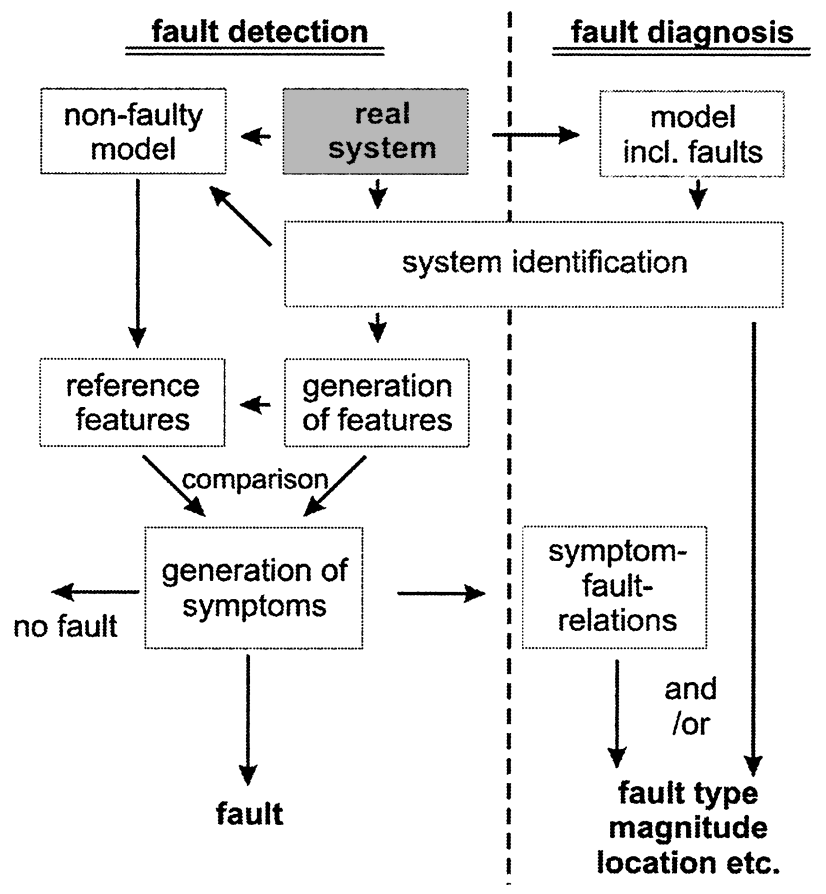

FIGURE 1

General concept of model-based diagnosis. 
the features of the non-faulty state and the actual state of the plant (Isermann, 1994). The comparison results in a statement that something is wrong and a fault occurred or everything is still alright.

The main steps of the fault diagnosis procedure are:

- Isolation: Determination of the faulty component

- Deriving symptom-fault relations and/or

- Modeling of possible faults with their effects

- Identification of fault magnitude, location, and reason

The isolation of a fault is relatively simple, due to the possibility of the AMBs to derive separated features based on the output $\hat{\mathbf{x}}$ input $\hat{\mathbf{f}}$, and system $\overline{\mathbf{H}}(\Omega)$ information. The main part of the diagnosis is to develop the relationships between the changes of the symptoms and the acting fault(s). One way in finding the most probable fault is to use inference mechanisms like fuzzylogic, classification methods like neural network algorithms, or a combination of both. These procedures are not covered within this article, as instead of using the change of symptom pattern to identify a possible fault, a model-aided diagnosis procedure is used. This method will be presented for an exemplary fault. The basic idea is to integrate different fault models into the updated non-faulty reference model, study their effects, and determine the parameters of the fault model to identify the most possible fault as well as its magnitude, location, etc.

\section{EXPERIMENTAL SETUP}

Within the presented research project, a magnetically suspended centrifugal pump serves as a representative for the turbomachines. The designed test rig is used to validate and demonstrate the performance of the developed model-based diagnosis.

The modular concept of the design enables an easy extension of a single-stage to four-stage pump system, both of which are subject for investigation. This article is restricted to the singlestage pump with respect to the experimental data. However, the developed method of the diagnosis procedure can be transferred to the four-stage pump as well as to any other turbomachine running in AMBs.

Figure 2 shows the scheme of the single-stage pump in AMBs. The pump is located between two active magnetic bearings levitating the rotor in five degrees of freedom. Besides the replacement of the conventional roller bearings through active magnetic bearings, the original pump system, especially the hydraulic part, remains unchanged.

In addition to the two mechanical seals sealing up the hydraulic part, the pump contains two contactless annular seals. One is placed at the suction side and one at the pressure side of the impeller, the latter being the balance piston. The rotor is coupled to the motor by a flexible membrane coupling. It should be mentioned that the dimensions of the magnetic bearings are largely oversized and not optimally designed for the pump.

Some technical data of the pump as well as of the AMB systems is presented in Table 1 . The entire test rig configuration is shown in Figure 3. The hydraulic periphery in which the pump

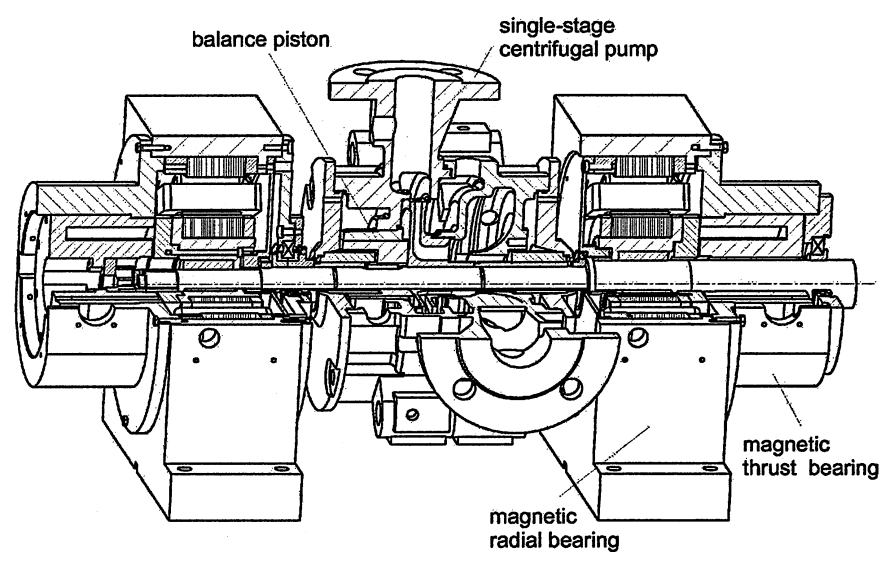

FIGURE 2

Scheme of the single-stage pump in AMBs.

is embedded is also demonstrated. It is an open circuit with the reservoir not shown. Water serves as process medium with a temperature kept constant at $20^{\circ} \mathrm{C}$.

\section{DATA ACQUISITION AND SIGNAL PROCESSING}

\section{Sensor Capability of the AMB System}

An important role in the development of the fault diagnosis relates to the precise measurement of the input-output signals (displacements and forces). The contactless measurement of displacements is state-of-the-art using inductive or eddy current sensors, whereas the contactless measurement of the forces acting on the rotor is more difficult. The latest results showed that with the integration of Hall probes into the air gap of radial AMBs (Knopf and Nordmann, 1998; Förch and Gähler, 1996) and of axial AMBs (Aenis and Nordmann, 2000), sufficient accuracy of the force measurement also can be gained.

The different force measurements in an AMB can be divided into two main groups. The first is based on the measurement

TABLE 1

Technical Data

\section{Pump data}

Rotational speed [rpm] 2950

Flow rate $\left[\mathrm{m}^{3} / \mathrm{h}\right] \quad 18$

Head [m] 21

Radial clearance piston seal [mm] $\quad 0.09$

Radial clearance impeller seal [mm] 0.12

\begin{tabular}{lcc}
\hline & Radial & Axial \\
\hline Magnetic Bearing Data & & \\
Air gap [mm] & .3 & 1.2 \\
AMB Force (per axis)[N] & 750 & 2200 \\
Premagnetisation, current [A] & 4.0 & 3.6 \\
Windings per pole pair [-] & 306 & 286 \\
Cross section area of pole $\left[\mathrm{mm}^{2}\right]$ & 864 & 2720 \\
\hline
\end{tabular}




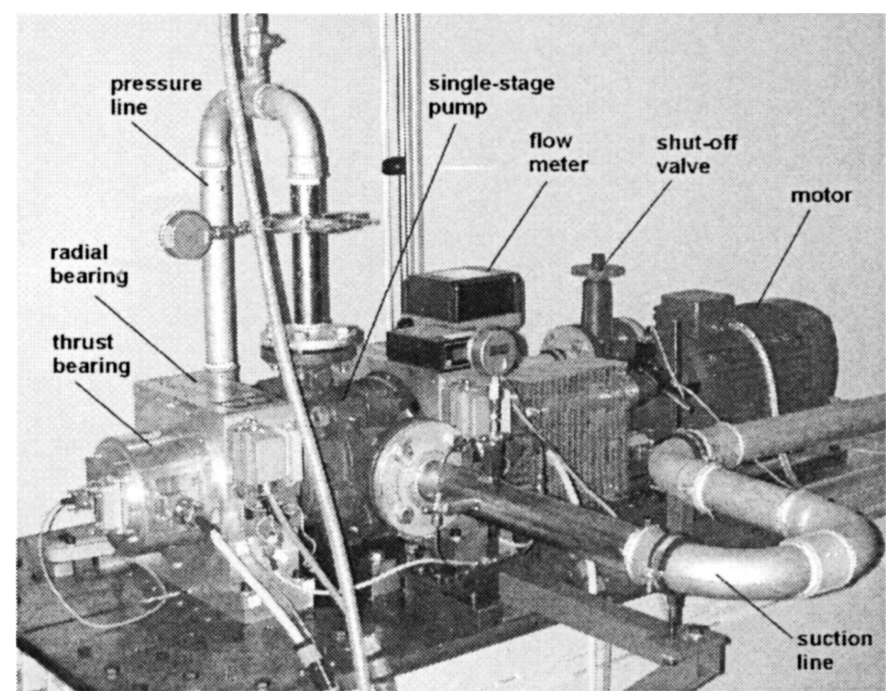

FIGURE 3

The test rig of the single-stage pump.

of coil currents and rotor displacements $(i-s$-method, reluctance network model) and the second method uses the direct measurement of the magnetic flux density $\overline{\mathbf{B}}_{k}$ with a Hall sensor at each pole $k$. The magnetic bearing force is then computed from

$$
\overline{\mathbf{f}}_{\mathrm{AMB}}=\frac{A_{\text {pole }}}{2 \mu_{0}} \sum_{k=1}^{8}\left|\overrightarrow{\mathbf{B}}_{k}\right| \overrightarrow{\mathbf{B}}_{k}
$$

with $A_{\text {pole }}$ being the cross-section area of the poles and $\mu_{0}$ the permeability of vacuum. The drawback is that the air gap has to be enlarged to integrate the Hall probes resulting in a decrease of load capacity of the bearing. To partially overcome this problem, a modified force measurement was presented by Förch and Gähler (1996), where only the north poles were equipped with Hall probes while the fluxes at the south poles were computed using an on-line approximation. This is the method used in the presented test rig. If a direct measurement of the flux density is not possible or desired, the so-called $i$-s-method can be applied, where the force is calculated using a linearized current-displacement relationship (Schweitzer et al., 1994)

$$
f_{x}=k_{i} i_{x}+k_{s} s_{x}
$$

where $i_{x}$ is the control current and $s_{x}$ the rotor displacement in the $x$-direction. The constants $k_{i}, k_{s}$ depend on the chosen design point of the magnetic bearings (bias current $i_{0}$ and air gap $s_{0}$ ). A more advanced force measurement based on coil currents and rotor displacements is a reluctance network model. The network model accounts much better for eccentric rotor positions and cross-coupling than the $i$-s-method. Depending on the requirements, the model can further be extended to consider leakage and fringing effects, eddy currents, nonlinear material behavior, hysteresis, etc., as it is described, for example, in Meeker et al. (1996) and Springer et al. (1998).
TABLE 2

Accuracies of Different Force Measurement Methods

\begin{tabular}{lcc}
\hline & $\begin{array}{c}\text { Centric rotor } \\
\text { range: } \pm \text { max. } \\
\text { bearing force }(\%)\end{array}$ & $\begin{array}{c}\text { Eccentric rotor } \\
\text { range: } \pm \text { max. } \\
\text { bearing force }(\%)\end{array}$ \\
\hline Radial bearing & & \\
$i$-s-method & 9 & 9 \\
Reluctance network & 8 & 7 \\
$\begin{array}{c}\text { 4 Hall sensors }+ \\
\text { approx. }\end{array}$ & 2 & 3 \\
$\begin{array}{c}\text { 8 Hall sensors } \\
\text { Axial bearing } \\
i-s \text {-method }\end{array}$ & $<1$ & 3 \\
Hall sensors & $32(1.5)^{*}$ & $34(8)^{*}$ \\
\hline
\end{tabular}

${ }^{*}$ When force range is restricted to $50 \%$ of maximum bearing force.

A detailed comparison of the achievable accuracies of the force measurement methods for different operating ranges can be found in Aenis and Nordmann (1999 and 2000). A short summary of the achievable accuracies is listed in Table 2.

\section{Actuator Capability of the AMB System}

The AMB system is digitally controlled and hence offers a great flexibility. With an onboard sine wave generator, the AMB system can be excited during regular operation with defined frequencies from $0-1 \mathrm{kHz}$. The performance of a step sine procedure and sequential excitation of all 5 inputs of the system with 5 linear independent force excitation patterns leads to 25 measurable transfer functions:

$$
\left[\begin{array}{c}
\hat{\mathbf{x}}_{A X} \\
\hat{\mathbf{x}}_{A y} \\
\hat{\mathbf{x}}_{B x} \\
\hat{\mathbf{x}}_{B y} \\
\hat{\mathbf{x}}_{Z}
\end{array}\right]=\left[\begin{array}{lllll}
\overline{\mathbf{H}}_{11} & \overline{\mathbf{H}}_{12} & \overline{\mathbf{H}}_{13} & \overline{\mathbf{H}}_{14} & \overline{\mathbf{H}}_{15} \\
\overline{\mathbf{H}}_{21} & \overline{\mathbf{H}}_{22} & \overline{\mathbf{H}}_{23} & \overline{\mathbf{H}}_{24} & \overline{\mathbf{H}}_{25} \\
\overline{\mathbf{H}}_{31} & \overline{\mathbf{H}}_{32} & \overline{\mathbf{H}}_{33} & \overline{\mathbf{H}}_{34} & \overline{\mathbf{H}}_{35} \\
\overline{\mathbf{H}}_{41} & \overline{\mathbf{H}}_{42} & \overline{\mathbf{H}}_{43} & \overline{\mathbf{H}}_{44} & \overline{\mathbf{H}}_{45} \\
\overline{\mathbf{H}}_{51} & \overline{\mathbf{H}}_{52} & \overline{\mathbf{H}}_{53} & \overline{\mathbf{H}}_{54} & \overline{\mathbf{H}}_{55}
\end{array}\right]\left[\begin{array}{c}
\hat{\mathbf{f}}_{A x} \\
\hat{\mathbf{f}}_{A y} \\
\hat{\mathbf{f}}_{B x} \\
\hat{\mathbf{f}}_{B y} \\
\hat{\mathbf{f}}_{Z}
\end{array}\right]
$$

with A, B standing for the 2 different radial bearings, and $x, y$ specifying the radial axes, respectively, and $z$ the axial axes (see Figure 4). It should be mentioned that not only are the frequency response functions of the rotor of the plant recordable, but also the different closed loop transfer functions are measurable, such as the sensitivity function. These functions enable us to evaluate the performance of the controller, which might be important to rate the tendency of a fault to destabilize the system.

\section{MODELING OF THE NON-FAULTY PLANT}

It is obvious that one step of the model-based diagnosis is the derivation of a parametric model of the plant. This is usually not a trivial process and may not justify the extra effort it requires for all diagnosis procedures. However, as mentioned above, the 


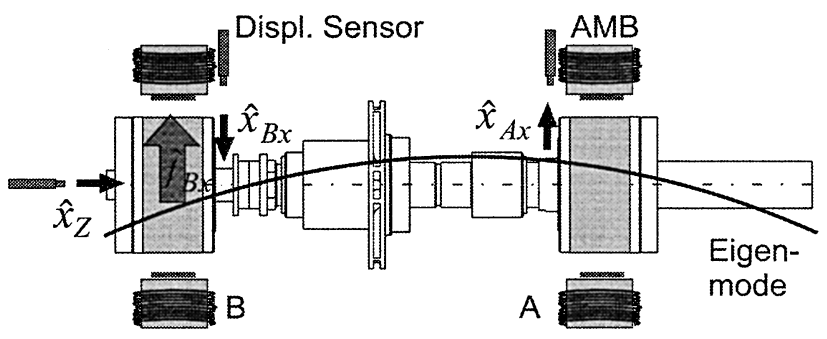

FIGURE 4

Actuator and sensor locations in the $x$-z-plane.

pump is part of the active magnetic bearing system and unlike conventional bearing systems, a controller is necessary to stabilize the unstable suspense state of the rotor, caused by the magnetic field. In recent years, especially for digital controlled bearings, the controller is mainly designed using model-based methods. That is exclusively the case the more the rotor has an elastic rotordynamic behavior, which is usually the case for multi-stage pump systems, where the impeller is placed between the bearings. Hence, a result of the controller design is the nonfaulty model of the plant as it is a part of the entire model of the mechatronic system. In summary, the modeling of the plant on one hand enables the design of a controller with high performance and on the other hand, enables a more detailed diagnosis.

The rotor of the pump is modeled through a finite element (FE) model (see Figure 5). The model update of the plain mechanical structure is performed by an experimental modal analysis to assure an accurate description of the reality. The detailed FE modeling was necessary to account for the dynamics of the entirely assembled rotor including several contact faces, strengthening of rotor because of shaft nut, etc. In a second step, the detailed FE-model is transferred to a beam model (see

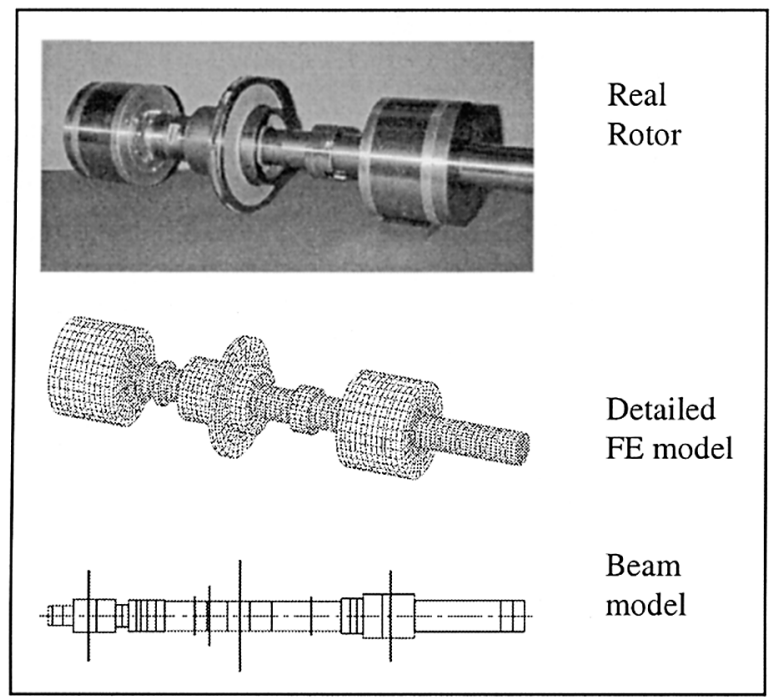

FIGURE 5

Real rotor and its FE-models.
Figure 5) to add the gyroscopic effects. The model reduction is simply performed by modal truncation. The rigid body modes as well as the first four bending modes are further considered. Modal damping is used to model the structural damping and a value of $0.75 \%$ was arbitrarily chosen.

Special routines are available as finite difference codes to consider the fluid forces acting on the rotor in the seals (impeller, balance piston), expressed as rotordynamic coefficients. In a similar way, the influence of the mechanical seals is added to the model. Finally, the dynamic of the housing is identified by an experimental modal analysis and its resonances are connected in parallel as single mass-spring-dampers. The radial degrees of freedom are coupled due to gyroscopic and fluid-structureinteraction effects. The axial degree of freedom is decoupled and consequently modeled separately.

Figure 6 shows a comparison between the measured frequency response function $\overline{\mathbf{H}}_{11}$ (see Equation (5)) of the nonfaulty pump rotor (thick curve), which serves as the reference, and the calculated frequency response function of the non-faulty model (thin curve). The high quality of the model is clearly demonstrated. The same holds true for the axial model, which is compared with the measured transfer function $\overline{\mathbf{H}}_{55}$ in Figure 7.

Once such a good model state is reached, the modal parameter, i.e., the eigenfrequencies $\omega_{0}$, the damping ratio $D$, as well as the eigenmodes can be computed from the finite element model for the non-faulty system and are listed in Table 3 in the last two columns. The resonance of the second radial rigid body mode as well as the second and third radial bending modes are clearly detectable in Figure 6, whereas the first bending mode can hardly be observed, due to the position of a vibration node in the vicinity of the sensor. It can further be observed that
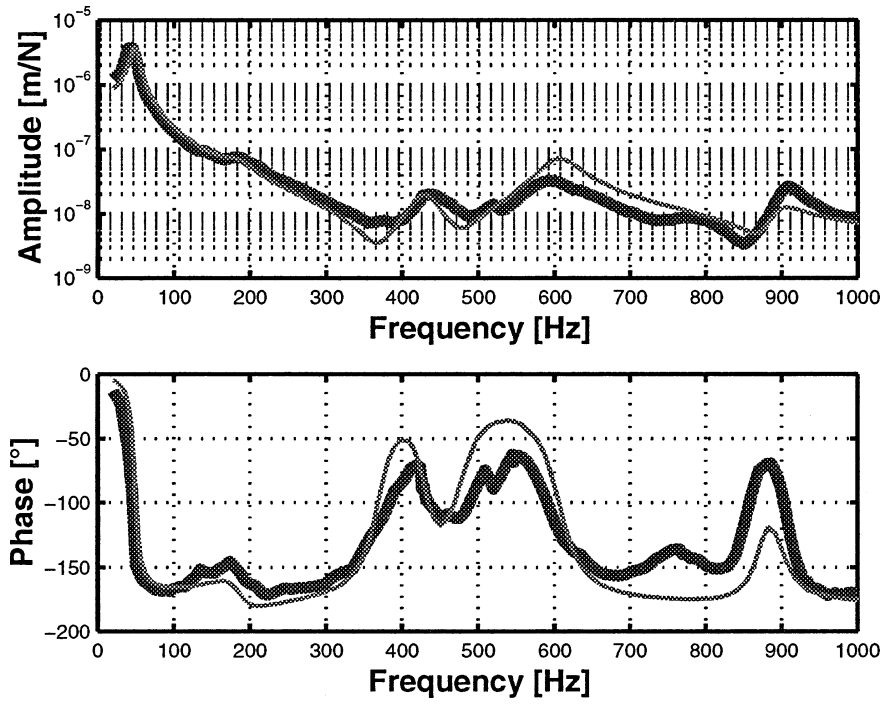

FIGURE 6

Measured (thick) and modeled (thin) radial frequency response function $\mathbf{H}_{11}$. 
TABLE 3

Reference Modal Parameters of the Non-Faulty System

\begin{tabular}{|c|c|c|c|c|}
\hline \multirow[b]{2}{*}{ Eigenmodes [-] } & \multicolumn{2}{|c|}{ Identified model } & \multicolumn{2}{|c|}{ Physical model } \\
\hline & Eigen freq. $\omega_{0}[\mathrm{~Hz}]$ & Damping ratio $D[\%]$ & Eigen freq. $\omega_{0}[\mathrm{~Hz}]$ & Damping ratio $D[\%]$ \\
\hline Radial: 1. Rigid body & \multicolumn{2}{|c|}{ Not detected } & 23 & 100.0 \\
\hline Axial: Rigid body & 37 & 45.9 & 34 & 23.0 \\
\hline Radial: 2. Rigid body & 43 & 10.4 & 49 & 8.9 \\
\hline Radial: 1. Bending & 184 & 12.4 & 186 & 10.6 \\
\hline Axial: Housing A & 273 & 3.0 & \multicolumn{2}{|c|}{ Not modeled } \\
\hline Axial: Housing B & 380 & 9.9 & \multicolumn{2}{|c|}{ Not modeled } \\
\hline Radial: Housing A & 435 & 4.8 & 430 & 4.0 \\
\hline Radial: 2. Bending & 593 & 5.4 & 606 & 3.1 \\
\hline Radial: 3. Bending & 778 & 3.8 & 895 & 1.5 \\
\hline Radial: Housing A/B? & 906 & 1.7 & \multicolumn{2}{|c|}{ Not modeled } \\
\hline
\end{tabular}

the water surrounding the rotor adds remarkable damping to the system. For the axial direction, the transfer function confirms the modeling of a single mass-spring-damper, representing the rotor axially supported in the mechanical seals. The resonances of the housing in the radial as well as in the axial direction are also detectable.

\section{GENERATION OF (REFERENCE) FEATURES}

There are four different methods of generating features, respectively, symptoms in model-based fault detection (Gertler, 1998): Kalman filter, diagnostic observer, parity relations, and parameter estimation. This article focuses on the generation
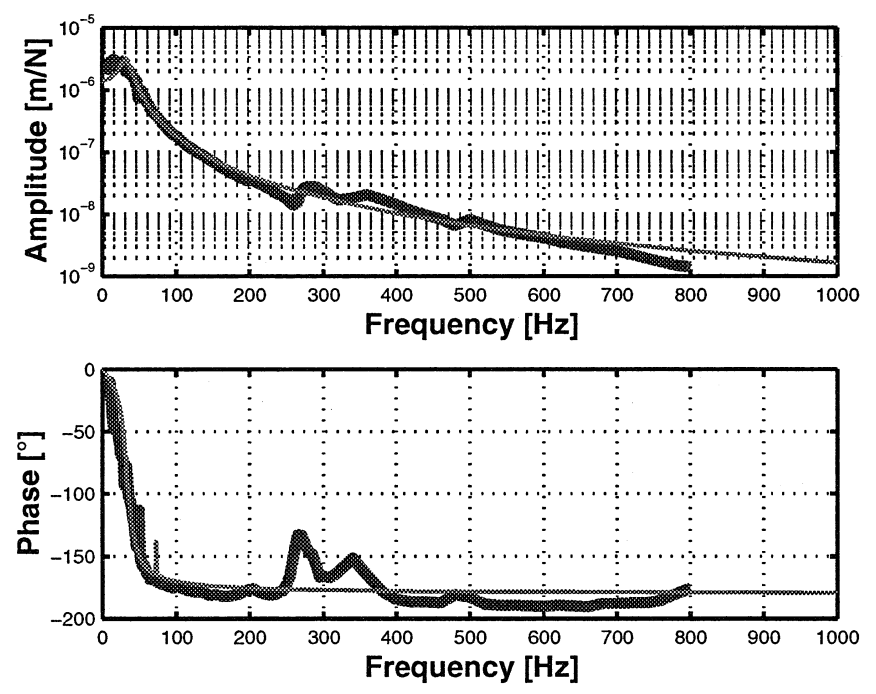

FIGURE 7

Measured (thick) and modeled (thin) axial frequency response function $\mathbf{H}_{55}$. of features from the measured frequency response functions, characterizing the system behavior. Therefore the parameter estimation is very suitable. The parameters, which have to be identified, are coefficients of a matrix fraction description, which reduces to a simple numerator/denominator representation for Single Input Single Output (SISO) models:

$$
\overline{\mathbf{H}}(s)=\mathbf{A}(s)^{-1} \mathbf{B}(s)=\frac{b_{0}+b_{1} s+b_{2} s^{2}+\cdots}{a_{0}+a_{1} s+a_{2} s^{2}+\cdots}
$$

The software used for identification is the FREQID-toolbox written by de Callafon and Van den Hof from Delft University of Technology. The estimation of the model is a least square curve fitting routine based on frequency domain data. For a more detailed discussion on the procedure, one is referred to de Callafon et al. (1996).

Figure 8 shows the result of the curve fitting of the frequency response function of the non-faulty pump. The thicker dark curve again represents the measured transfer function $\overline{\mathbf{H}}_{11}$. The lighter curve represents the identified SISO-system model, with a model order of 14 . The high quality of the fitted model is demonstrated. With the identified model, a second set of modal parameters (i.e., poles of Equation (6)) can be computed, which are listed in the second and third column of reference in Table 3. It should be mentioned that here only the eigenfrequencies and the damping ratio can be extracted because of the insufficient information receiving from two sensor planes with respect to the reconstruction of the mode shapes. Figure 9 shows the comparison of the measured and identified axial frequency response function $\overline{\mathbf{H}}_{55}$. The calculated modal parameters based on the identified model are listed in Table 3 and complete the reference matrix. The advantage of the model-based procedure is demonstrated by the first column of Table 3. Without the knowledge added from the physical model, it would be impossible to distinguish and classify the different eigenfrequencies. 

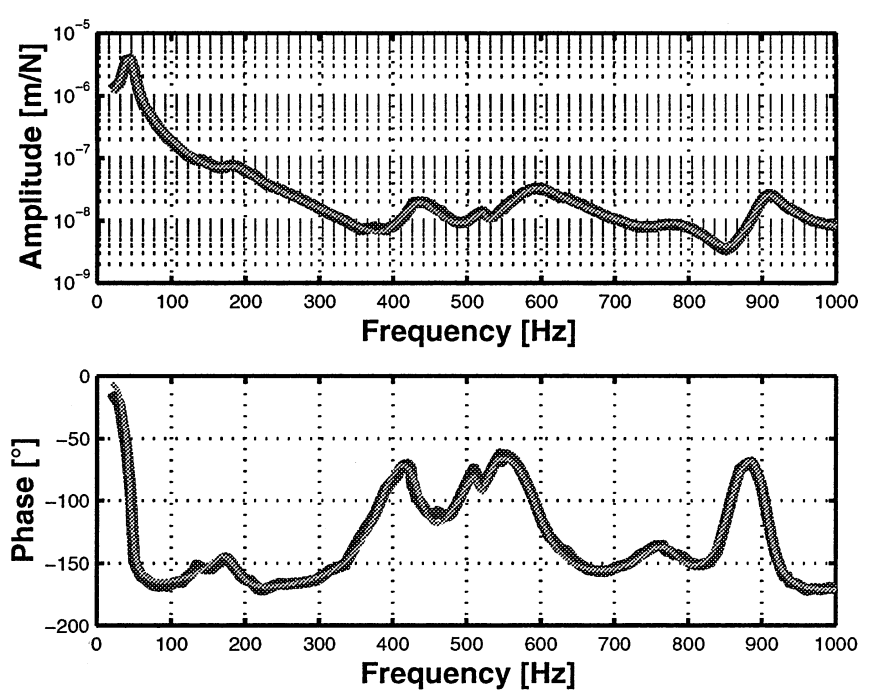

FIGURE 8

Measured (dark) and fitted (light) radial frequency response function $\mathbf{H}_{11}$.

\section{AN EXEMPLARY FAULT}

The diagnosis procedure developed using frequency domain data received from the AMBs is demonstrated by an exemplary fault. As mentioned earlier, one assumes a fault, which alters the system $\overline{\mathbf{H}}(\Omega)$. Analogous to the deriving of the reference features of the non-faulty system, features (i.e., modal parameters) of the faulty system are identified. Figure 10 shows such a measured transfer function (dark curve) of the faulty system together with the identified model (light curve). Computing the modal parameters from the identified model leads to the values listed in the last two columns of Table 4. For an easy comparison, the reference modal parameters from the radial transfer function of Table 3 are repeated in the first columns. This comparison
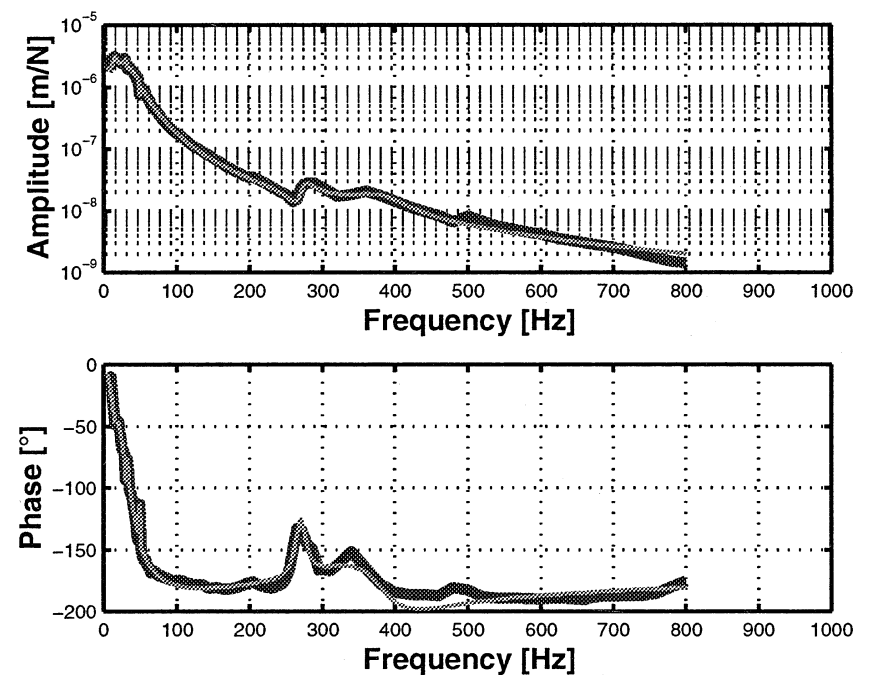

FIGURE 9

Measured (dark) and fitted (light) axial response function $\mathbf{H}_{55}$.
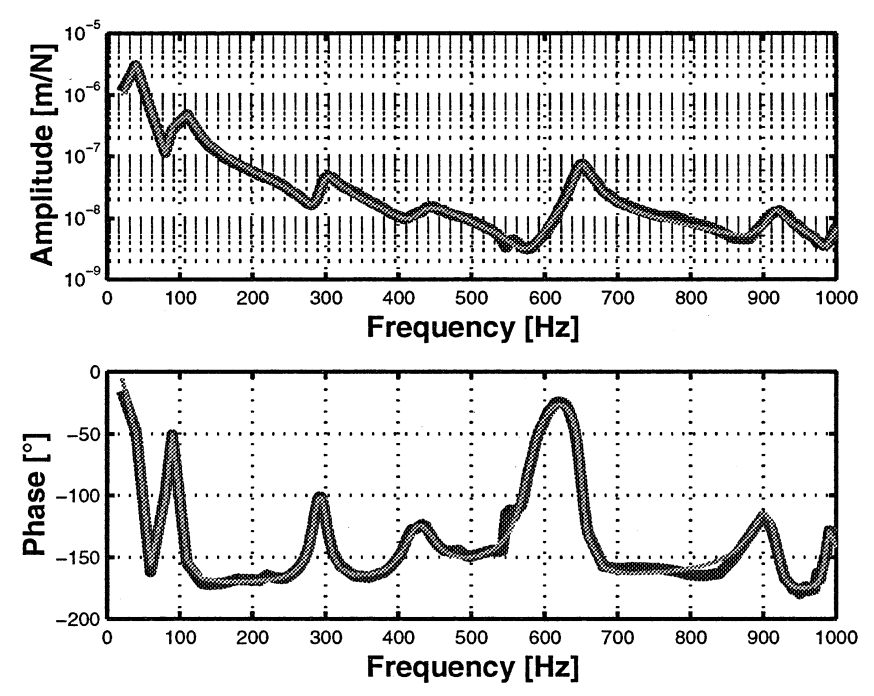

FIGURE 10

Measured (dark) and fitted (light) radial frequency response function $\mathbf{H}_{11}$ of a faulty configuration.

of the features generates symptoms for the faulty system, which confirms that something is going wrong. The result of the fault detection is positive. A few facts are already recognizable from the table. The resonance of Housing A is unchanged, hence something is wrong in the rotordynamic system. Furthermore, all eigenfrequencies, especially the bolded ones, are shifted to higher frequencies and the damping ratio is significantly decreased.

The problem of the second step of the diagnosis part is to identify the acting fault. This can be done by evaluating the specific symptom pattern by using symptom-fault relations or by a model-based procedure, which is used here. The non-faulty
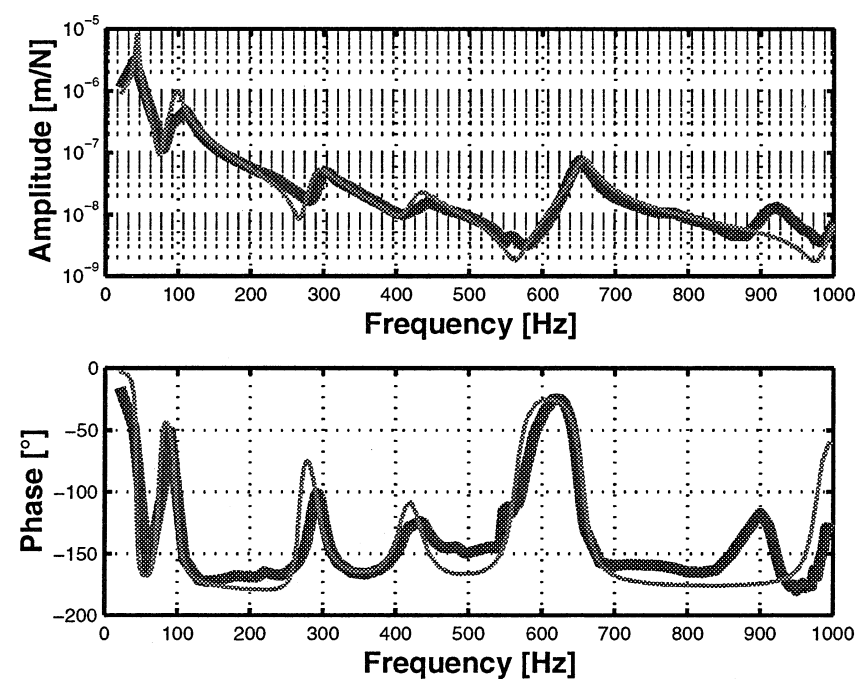

FIGURE 11

Measured (thick) and modeled (thin) radial frequency response function $\mathbf{H}_{11}$ of a faulty configuration. 
TABLE 4

Comparison between the Features of the Reference and a Faulty Case

\begin{tabular}{|c|c|c|c|c|}
\hline \multirow[b]{2}{*}{ Eigenmodes [-] } & \multicolumn{2}{|c|}{ Reference model } & \multicolumn{2}{|c|}{ Actual model } \\
\hline & Eigen freq. $\omega_{0}[\mathrm{~Hz}]$ & Damping ratio $D[\%]$ & Eigen freq. $\omega_{0}[\mathrm{~Hz}]$ & Damping ratio $D[\%]$ \\
\hline 1. Rigid body & \multicolumn{2}{|c|}{ Not detected } & 43 & 9.5 \\
\hline 2. Rigid body & 43 & 10.4 & 100 & 5.9 \\
\hline 1. Bending & 184 & 12.4 & 301 & 3.4 \\
\hline Housing A & 435 & 4.8 & 437 & 6.0 \\
\hline 2. Bending & 593 & 5.4 & 651 & 1.3 \\
\hline 3. Bending & 778 & 3.8 & 993 & 1.0 \\
\hline Housing A/B? & 906 & 1.7 & 916 & 1.3 \\
\hline
\end{tabular}

physical model is extended through different fault models, one of which is the cancellation of the fluid-structure-interaction parameters, representing a dry run. Evaluating the corresponding frequency response functions and comparing the simulated faulty ones with the measured ones leads to the identification of the possible fault: dry run. Figure 11 shows the comparison between the measured faulty transfer function and the simulated fault. A good agreement up to $800 \mathrm{~Hz}$ can be recognized and confirms the diagnosis.

\section{CONCLUSION}

The procedure of model-based diagnosis for turbomachines running in active magnetic bearings has been described. A magnetically suspended centrifugal pump has been chosen as the application to demonstrate the developed routines. In particular, the special capabilities of an AMB system like online force and frequency response function measurements have been introduced. Furthermore, a high quality model of the rotordynamic system including the fluid-structure-interaction in the seals for the model-based diagnosis has been described. The focus laid on the identification of the modal parameters to generate features, respectively symptoms to detect faulty conditions which are altering the system, i.e., the mass, damping, and stiffness matrices. Using this procedure and the updated model, a dry-run condition of the pump has been detected and diagnosed. The future development concentrates on the development of automatic diagnosis procedures and the integration of other faults like wear of the balance piston, crack in the rotor, or loosening of a shaft nut, some of which have already been detected Strassburger et al. (2001). Furthermore, for a practical use of the developed modelbased diagnosis not only the modal parameters should be used as features, but also information gained from the bearing forces. Then faults like misalignment, unbalance, or even part-load and overload conditions are detectable (Strassburger et al., 2001). It should be mentioned that this model-based diagnosis using AMBs not only works for centrifugal pumps but for all turbomachines running in AMBs. Hence, turbomachines equipped with such integrated model-based diagnosis lead to machines with higher performance, higher reliability, and longer lifetimes.

\section{ACKNOWLEDGMENTS}

This article is a result from the work of the Special Research Program (SFB 241) sponsored by the German Research Council (DFG).

\section{REFERENCES}

Aenis, M., and Nordmann, R. 2000. Active Magnetic Bearings for Fault Detection in a Centrifugal Pump, 7th International Symposium on Magnetic Bearings. Zurich, Switzerland.

Aenis, M., and Nordmann, R. 1999. A Precise Force Measurement in Magnetic Bearings for Diagnosis Purposes, 5th International Symposium on Magnetic Suspension Technology. Santa Barbara, California.

Allaire, P. E., Imlach, J., McDonald, J. P., Humphris, R. R., Lewis, D. W., Banerjee, B. B., Blair, B. J., Claydon, J., and Flack, R. D. 1989. Design, Construction and Test of Magnetic Bearings in an Industrial Canned Motor Pump, 6th International Pump Users Symposium. Houston, Texas.

Baun, D. O., and Flack, R. D. 1997. A Plexiglas Research Pump with Calibrated Magnetic Bearings/Load Cells For Radial and Axial Hydraulic Force Measurement, ASME Fluids Engineering Division Summer Meeting, FEDSM'97.

de Callafon, R. A., Roover, D., and Van den Hof, P. M. J. 1996. Multivariable Least Squares Frequency Domain Identification using Polynomial Matrix Fraction Description, Proceedings of the 36th IEEE Conference on Decision and Control.

Förch, P., and Gähler, C. 1996. AMB System for Rotordynamic Experiments: Calibration Results and Control, 5th International Symposium on Magnetic Bearings. Kanazawa.

Gertler, J. 1998. Fault Detection and Diagnosis in Engineering Systems. Marcel Dekker, Inc., New York.

Gopalakrishan, S. 1999. Pump research and development: Past, present, and future. Transactions of the ASME, Journal of Fluids Engineering 121.

Guinzburg, A., and Buse, F. W. 1994. Axial and Radial Forces on a Pump Impeller Obtained with a Magnetic Bearing Force Measurement Rig, 4th International Symposium on Magnetic Bearings. Zurich, Switzerland.

Hergt, P. H. 1999. Pump research and development: Past, present, and future. Transactions of the ASME, Journal of Fluids Engineering 121. 
Humphris, R. R. 1992. A Device for Generating Diagnostic Information for Rotating Machinery Using Magnetic Bearings, in $M A G$ '92, Industrial Conference and Exhibition on Magnetic Bearings. Alexandria Virginia.

Isermann, R. (Hrsg.) 1994. Ueberwachung und FehlerdiagnoseModerne Methoden und ihre Anwendungen bei technischen Systemen, VDI-Verlag. Duesseldorf, Germany.

ISMB. 2000. Proceedings of the 7th International Symposium on Magnetic Bearings, Zurich, Switzerland.

Knopf, E., and Nordmann, R. 2001. Identification of the Dynamic Characteristics of Turbulent Journal Bearings, 8th ISROMAC. Honolulu, Hawaii.

Knopf, E., and Nordmann, R. 1998. Active Magnetic Bearings for the Identification of Dynamic Characteristics of Fluid Bearings, 6th International Symposium on Magnetic Bearings. Cambridge, Massachusetts, USA.

McGinnis, G., Cooper, P., Janik, G., Jones, G., and Shultz, R. 1990. Application of Magnetic Bearings in a Multistage Boiler Feed Pump, 2nd International Symposium on Magnetic Bearings. Tokyo, Japan.
Meeker, D. C., Maslen, E. H., and Myounggyu, D. N. 1996. A Wide Bandwidth Model for the Electrical Impedance of Magnetic Bearings, NASA conference publication No. 3336/ PT2.

Natke, H. G., and Cempel, C. 1997. Model-Aided Diagnosis of Mechanical Systems. Springer, Berlin Heidelberg.

Pottie, K., Wallays, G., Verhoeven, J., Sperry, R., Gielen, L., De Vis, D., Neumer, T., Matros, M., and Jayawant, R. 1994. Active Magnetic Bearings Used in BW/IP Centrifugal Pumps, 4th International Symposium on Magnetic Bearings. Zurich, Switzerland.

Schweitzer, G., Bleuler, H., and Traxler, A. 1994. Magnetlager. Springer, Berlin Heidelberg.

Springer, H., Schlager, G., and Platter, T. 1998. A Nonlinear Simulation Model for Active Magnetic Bearing Actuators, 6th International Symposium on Magnetic Bearings. Cambridge, Massachusetts, USA.

Strassburger, S., Aenis, M., and Nordmann, R. 2001. Magnetlager zur Schadensdiagnose und Prozessoptimierung, 5th. International Symposium SIRM. Vienna, Austria. 

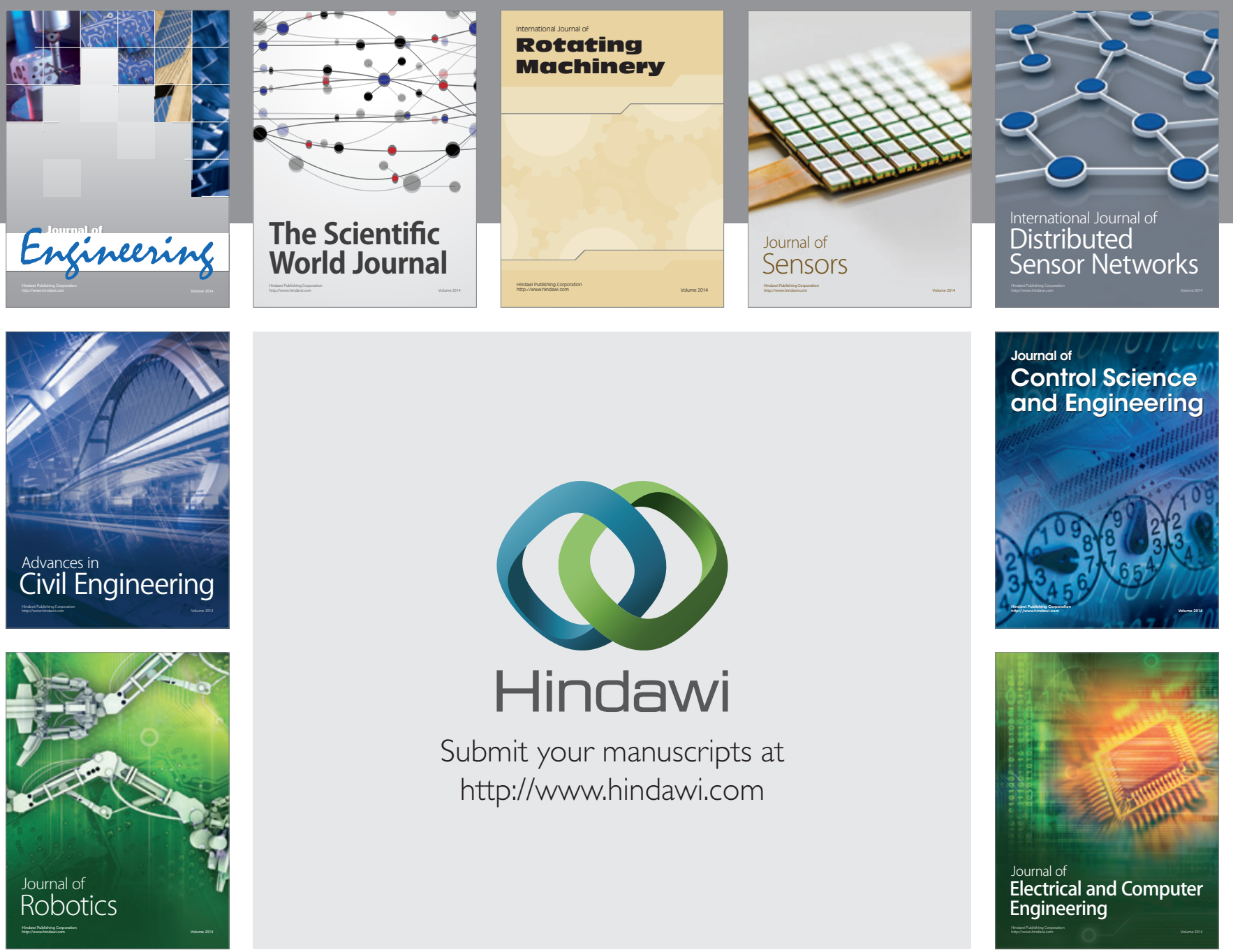

Submit your manuscripts at

http://www.hindawi.com
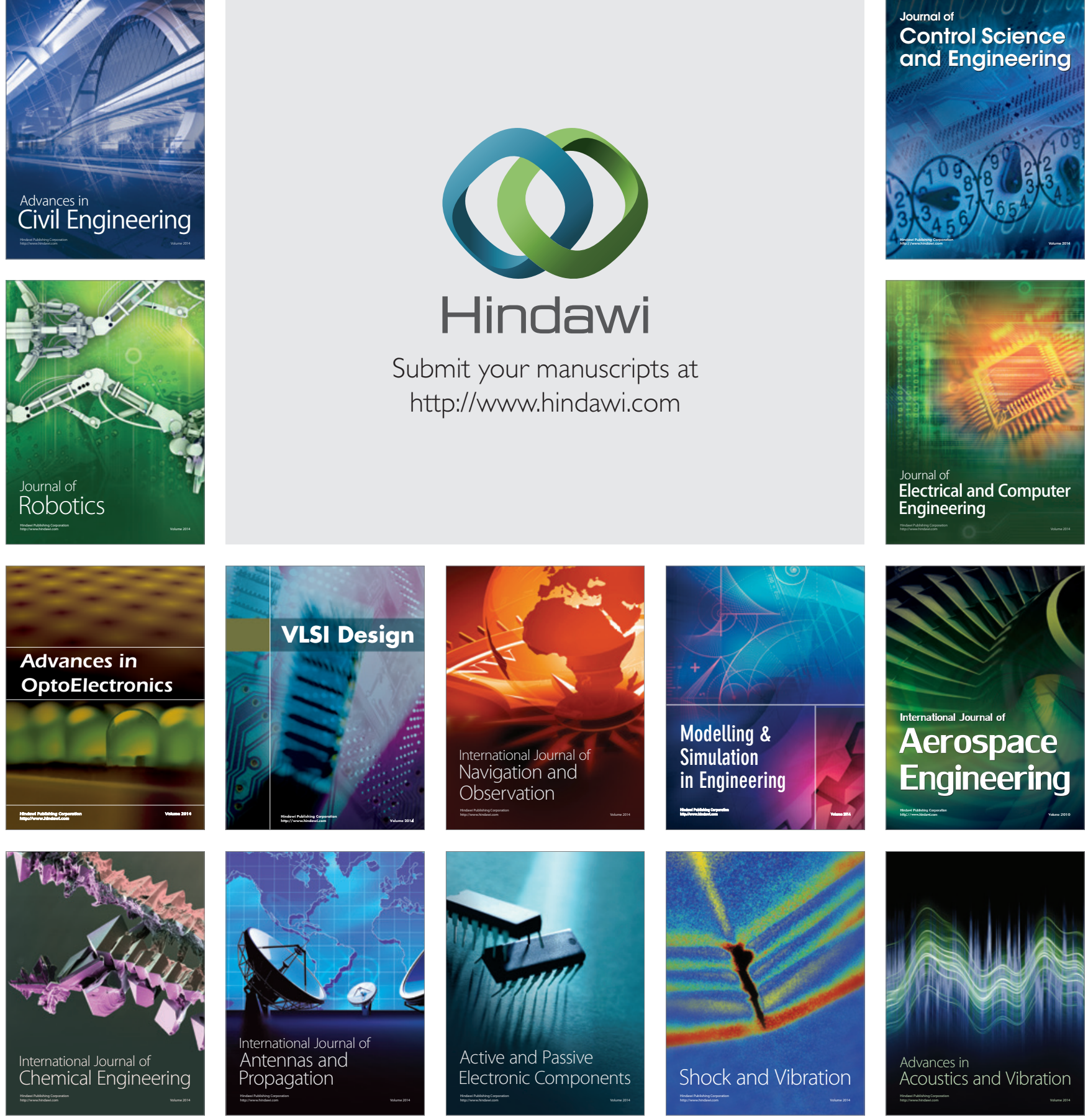